\title{
Prognostic Significance of Pancreatic Metastases from Renal Cell Carcinoma in Patients Treated with Tyrosine Kinase Inhibitors
}

\author{
PAWEL CHROM, RAFAL STEC, LUBOMIR BODNAR and CEZARY SZCZYLIK \\ Department of Oncology, Military Institute of Medicine, Warsaw, Poland
}

\begin{abstract}
Aim: The study aimed to define the true impact of pancreatic metastases (PM) from renal cell carcinoma on overall survival (OS) in patients treated with first-line tyrosine kinase inhibitors. Patients and Methods: Overall, 321 consecutive patients were analysed. The influence of PM on OS was assessed using the Kaplan-Meier estimator and the logrank test (unadjusted and adjusted) and two multivariabe Cox proportional hazards regressions (CPHR). Results: Thirty-four patients (10\%) had PM and 287 (90\%) had sites of metastasis other than the pancreas; the median OS was 46.1 and 23.1 months, respectively (unadjusted log-rank $p=0.020$; adjusted log-rank $p=0.544)$. The PM status was an insignificant factor for $O S$ in both CPHR (hazard ratio $(H R)=0.84, p=0.603$, and $H R=0.66, p=0.098)$. Conclusion: The presence of PM was not an independent prognostic factor, but was rather an indicator of an indolent course of the disease.
\end{abstract}

Renal cell carcinoma (RCC) is the most frequent type of kidney cancer and accounts for $3 \%$ of malignancies in adults. About $20 \%$ of patients with RCC have metastases at the initial diagnosis and up to half of patients develop recurrence after complete resection of the primary tumour (1-4). Nowadays, these patients are treated with systemic molecular targeted therapies (MTT) that include tyrosine kinase inhibitors (TKI) as the standard of care in first-line therapy and subsequent lines (5-6). Despite the significant improvement in efficacy of this type of drug compared to cytokine-based regimens, the treatment remains palliative in

This article is freely accessible online.

Correspondence to: Pawel Chrom, Department of Oncology, Military Institute of Medicine, Szaserow 128 st, PO Box: 04141, Warsaw, Poland. Tel: +48 600057413, Fax: +48 226103098, e-mail: pawel.chrom@gmail.com

Key Words: Metastatic renal cell carcinoma, overall survival, pancreatic metastases, prognostic factor, tyrosine kinase inhibitor. nature, i.e. aims to maximally increase patient survival with having little curative potential. Moreover, there are still no factors possessing predictive value for MTT, thus patient evaluation and prognosis is made using selected baseline characteristics. The most commonly used and validated tool for survival prediction in patients with metastatic RCC is the International Metastatic Renal Cell Database Consortium (IMDC) model. It consists of six factors independently associated with poor outcome: Karnofsky performance status (KPS) less than $80 \%$, time from diagnosis to start of systemic treatment less than 12 months, haemoglobin level less than the lower limit of normal (LLN), serum corrected calcium level, neutrophil count and platelet count greater than the upper limit of normal (ULN). Patients are stratified into favourable-, intermediate- and poor-risk groups according to the total number of factors $(0$, 1-2 and 3-6, respectively) (7). Other researchers concluded that the involvement of the metastatic process in specific organs, e.g. liver or bone, as well as an increasing number of metastatic sites may be additional features of reduced survival $(8,9)$. This is obviously a rational concept that a higher burden of malignant disease usually results in faster progression and death. In contrast to this observation, a few studies reported that patients with pancreatic metastases (PM) lived significantly longer than patients with metastases at sites other than the pancreas (10-12). It was further suggested that the presence of PM may be considered as an independent factor of favourable prognosis (11-13). This means that PM may increase the probability of survival regardless of the status of other criteria known to have an impact on the prognosis (e.g. those included in the IMDC model). This interesting concept led us to investigate whether PM truly influences survival of patients with metastatic RCC or just act as an indicator of favourable prognosis that may be actually due to other factors. Because PM usually occurs a long time after initial RCC diagnosis (14), we also explored the association between PM and the interval between the diagnosis and initiation of first-line TKI to answer the research question. 


\section{Patients and Methods}

Patients. We performed a retrospective analysis of consecutive patients with metastatic RCC who had begun treatment with first-line TKI from November 2009 to March 2016 at the Department of Oncology, Military Institute of Medicine, Warsaw, Poland. Patients who: (a) had metastatic RCC of any histological subtype, (b) had undergone prior nephrectomy or nephron-sparing surgery, (c) were treated with TKI using standard schedules, (d) had no other primary malignancies, (e) had no adjuvant or investigational therapy, were eligible. Patients were assigned to the PM group if they had PM at the start of first-line TKI. All other patients, including those surgically and completely treated for PM before TKI initiation, were included in the non-PM group. Individual patient data were gathered from institutional medical records. The study was approved by the Ethics Committee of the participating centre (the study protocol number: 84/WIM/2016).

Outcome and statistical analysis. The assessed outcome was overall survival (OS) which was defined as the time from the initiation of first-line TKI to death from any cause. Descriptive statistics included the median and range for continuous variables as well as frequency and percentage for categorical variables. The differences in baseline features between the PM and non-PM groups were examined using the Mann-Whitney $U$-test for continuous variables and the Pearson chi-square or Fisher's exact test (in the case of no more than five expected frequencies in each cell of a studied contingency table) for categorical variables. The association between the PM status and the interval between diagnosis of RCC and TKI initiation was assessed using the Pearson chi-square (or Fisher's exact) test, Cramer's V and the odds ratio. The Kaplan-Meier method was used to estimate survival curves and medians with calculation of $95 \%$ confidence interval (CI) using the log-log transformation. The log-rank test was used to compare differences in survival probabilities between the PM and non-PM groups. The impact of PM on OS was examined using (a) adjusted Kaplan-Meier estimator and log-rank test based on inverse probability of treatment weighting (IPTW) that reduces the bias caused by unbalanced distribution of confounders (15), and (b) two procedures of the Cox proportional hazards regression (CPHR). In the first CPHR procedure, univariable analyses were performed on all collected variables of potential prognostic ability and those that reached statistical significance were included in a multivariable analysis. In the second CPHR procedure, the hazard ratio (HR) and its standard error for PM status were adjusted for the six IMDC criteria. To ascertain the strength of the relationship between assessed variables and OS, both CPHR procedures were repeated on 500 new datasets generated using bootstrap (random-sampling with replacement) method (16). The survival data were last updated on October 15, 2016. Patients alive on that date or lost to follow-up were censored. The Shemper and Smith method was used to estimate the median followup time (17). Patients with missing values for the PM status or the IMDC criteria were excluded from analyses. $p$-Values less than 0.05 (two-sided) indicated statistical significance for all tests. All computations were performed using Stata, version 14.2 (StataCorp, College Station, TX, USA) and R, version 3.2.5 (The R Foundation for Statistical Computing, Vienna, Austria).

\section{Results}

Three hundred and twenty-one patients were analysed after excluding five patients with missing values for serum- corrected calcium. Thirty-four (10\%) patients had metastatic involvement of the pancreas at first-line TKI initiation and were assigned to the PM group. There were four (1\%) patients included in the non-PM group with complete resection of PM before the start of systemic treatment. Median time from diagnosis to development of PM was 84 (range $=0-235$ ) months. Only one patient in the PM group $(1 / 34,3 \%)$ had metastasis to the pancreas at the initial diagnosis; in all remaining cases, PM were metachronous. Pancreas was the only metastatic site in two patients $(2 / 34$, $6 \%)$. Solitary metastasis to the pancreas accounted for 10 cases $(10 / 34,29 \%)$, whereas multiple pancreatic lesions were found in 24 patients $(24 / 34,71 \%)$. The PM and non-PM groups were in the majority similar for baseline characteristics (Table I). However, patients in the PM group had more frequent metastasis to the contralateral kidney and less frequent metastasis to bone, local recurrence and Fuhrman grade of 3-4. In addition, the time between the initial diagnosis and TKI initiation was significantly longer in the PM group, with the median almost eight-fold greater than in the non-PM group. The distribution of patients within the IMDC risk groups differed significantly between the two cohorts. The PM group was mostly represented by patients in the favourable-risk group, while patients in the non-PM group were distributed more equally with about half assigned to the intermediate-risk group.

In the non-PM group, the proportion of patients with a diagnosis-to-treatment interval less than 12 months was almost equal to the proportion of patients with an interval of 12 months or greater (52\% vs. $48 \%$, respectively). In the PM group, most patients (85\%) received treatment after one year from the diagnosis. The association between those two variables was statistically significant (Pearson chisquare $=16.56, p<0.001$, Cramer's V $=0.227, p<0.001)$. The odds of having first-line TKI initiation at 12 months or more from the diagnosis were 6.2-times (95\% CI=2.3-16.4, $p<0.001)$ greater in the PM group than in the non-PM group.

The median follow-up time was 55.5 (95\% CI=49.0-60.7) months. The median OS for the whole cohort of patients was 23.2 (95\% CI=19.9-28.0) months. There were 19 (56\%) deaths in the PM group and 200 (70\%) in the non-PM group. The median OS was 46.1 (95\% CI=20.8-not estimable) months for the PM group and 22.5 (95\% CI=18.7-25.9) months for the non-PM group. Patients in the PM group had significantly increased OS compared with patients in the non-PM group (unadjusted log-rank $p=0.020$, Figure 1A). However, the adjustment using IPTW revealed closer and crossing survival curves of the PM and non-PM groups (Figure 1B) and non-significant result of the log-rank test $(p=0.544)$.

In the first CPHR procedure, the presence of PM was amongst 20 factors associated with OS using univariable analysis $(\mathrm{HR}=0.58,95 \% \mathrm{CI}=0.36-0.92, p=0.022)$. However, 
Table I. Patients characteristics at the start of therapy with first-line tyrosine kinase inhibitor (TKI) (total number=321).

\begin{tabular}{|c|c|c|c|}
\hline Variable & $\mathrm{PM}$ group $(\mathrm{N}=34)$ & Non-PM group $(\mathrm{N}=287)$ & $p$-Value \\
\hline Age, years: median (range) & $63(43-78)$ & $61(22-85)$ & 0.405 \\
\hline Time from diagnosis to first-line TKI initiation, months: median (range) & $85(3-270)$ & $11(0-243)$ & $<0.001$ \\
\hline Time from diagnosis to first-line TKI initiation $<1$ year: $\mathrm{n}(\%)$ & $5(15)$ & $148(52)$ & $<0.001$ \\
\hline Male: $\mathrm{n}(\%)$ & $19(56)$ & $196(68)$ & 0.146 \\
\hline Primary tumour site, right: $\mathrm{n}(\%)$ & $17(50)$ & $137(48)$ & 0.803 \\
\hline Fuhrman grade, $3-4: \mathrm{n}(\%)$ & $4(17)^{\mathrm{a}}$ & $112(42)^{\mathrm{b}}$ & 0.019 \\
\hline Non-clear cell histology: $\mathrm{n}(\%)$ & $1(3)$ & $18(6)$ & 0.705 \\
\hline Sarcomatoid features: $\mathrm{n}(\%)$ & $0(0)$ & $18(6)$ & 0.235 \\
\hline Number of metastatic sites: median (range) & $3(1-7)$ & $2(1-6)$ & 0.021 \\
\hline Number of metastatic sites: $\mathrm{n}(\%)$ & & & 0.131 \\
\hline $1-2$ & $13(38)$ & $149(52)$ & \\
\hline$\geq 3$ & $21(62)$ & $138(48)$ & \\
\hline \multicolumn{4}{|l|}{ Metastatic sites: $\mathrm{n}(\%)$} \\
\hline Lung & $21(62)$ & $210(73)$ & 0.162 \\
\hline Lymph nodes & $13(38)$ & $140(49)$ & 0.244 \\
\hline Bone & $3(9)$ & $93(32)$ & 0.005 \\
\hline Liver & $10(29)$ & $58(20)$ & 0.214 \\
\hline Suprarenal gland & $8(24)$ & $45(16)$ & 0.244 \\
\hline Brain & $1(3)$ & $15(5)$ & 0.999 \\
\hline Local recurrence & $3(9)$ & $74(26)$ & 0.029 \\
\hline Contralateral kidney & $8(24)$ & $18(6)$ & 0.003 \\
\hline KPS: n (\%) & & & 0.832 \\
\hline 100 & $14(41)$ & $111(39)$ & \\
\hline $80-90$ & $20(59)$ & $167(58)$ & \\
\hline$<80$ & $0(0)$ & $9(3)$ & \\
\hline Haemoglobin <LLN: n $(\%)$ & $3(9)$ & $59(21)$ & 0.101 \\
\hline Corrected calcium >ULN: $\mathrm{n}(\%)$ & $3(9)$ & $32(11)$ & 0.681 \\
\hline $\mathrm{LDH}>\mathrm{ULN}: \mathrm{n}(\%)$ & $8(25)^{\mathrm{c}}$ & $74(27)^{d}$ & 0.853 \\
\hline Serum albumin <LLN: n $(\%)$ & $2(6)$ & $38(14)$ & 0.206 \\
\hline Neutrophil count $>$ ULN: $\mathrm{n}(\%)$ & $3(9)$ & $23(8)$ & 0.746 \\
\hline Platelet count >ULN: $\mathrm{n}(\%)$ & $2(6)$ & $42(15)$ & 0.196 \\
\hline NLR $\geq 3.6: \mathrm{n}(\%)$ & $13(38)$ & $96(33)$ & 0.577 \\
\hline PLR $\geq 157: \mathrm{n}(\%)$ & $14(42)$ & $155(54)$ & 0.157 \\
\hline IMDC risk group: $\mathrm{n}(\%)$ & & & $<0.001$ \\
\hline Favourable & $24(71)$ & $95(33)$ & \\
\hline Intermediate & $9(26)$ & $158(55)$ & \\
\hline Poor & $1(3)$ & $34(12)$ & \\
\hline Prior immunotherapy: n (\%) & $6(18)$ & $29(10)$ & 0.238 \\
\hline First-line TKI therapy: $\mathrm{n}(\%)$ & & & 0.536 \\
\hline Sunitinib & $24(70)$ & $216(75)$ & \\
\hline Sorafenib & $4(12)$ & $20(7)$ & \\
\hline Pazopanib & $6(18)$ & $51(18)$ & \\
\hline Second-line systemic therapy: n (\%) & $12(35)$ & $131(46)$ & 0.251 \\
\hline
\end{tabular}

IMDC, International Metastatic Renal Cell Carcinoma Database Consortium; KPS: Karnofsky performance status; LDH, lactate dehydrogenase; LLN, lower limit of normal; NLR: neutrophil-to-lymphocyte ratio; PLR: platelet-to-lymphocyte ratio; PM, pancreatic metastases; ULN, upper limit of normal. Number of patients evaluated: ${ }^{a} 23,{ }_{2} 64,{ }^{c} 32,{ }^{2} 279$.

after fitting the multivariable model, PM was no longer a significant factor for survival $(\mathrm{HR}=0.84,95 \% \mathrm{CI}=0.44-1.62$, $p=0.603$ ). Factors independently affecting the outcome by increasing the hazard of death were: KPS $<80 \%$, Fuhrman grade 3-4, presence of lung metastases, haemoglobin less than the LLN, neutrophil-to-lymphocyte ratio $\geq 3.6$, serum lactate dehydrogenase greater than the ULN, and serum albumin less than the LLN (Table II). In the second CPHR procedure, the PM status did not reach statistical significance after adjusting for the six IMDC criteria (HR=0.66, 95\% CI $=0.40-1.08, p=0.098$, Table III). The bootstrap validation strengthened the conclusion of a nonsignificant influence of the PM status on OS in both CPHR procedures (Tables II and III). 
A

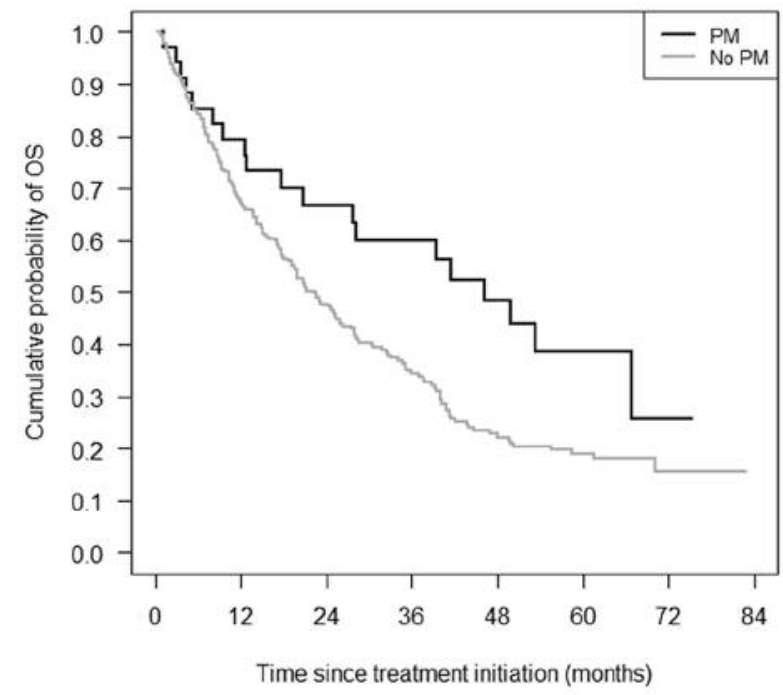

B

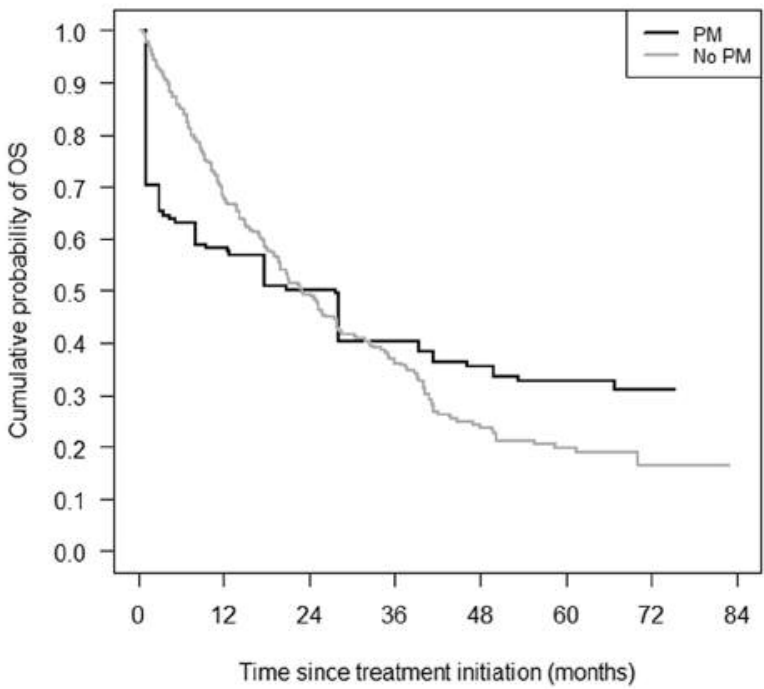

Figure 1. Unadjusted (A), and adjusted (B) Kaplan-Meier estimators for overall survival (OS) stratified by the presence of pancreatic metastases (PM).

Table II. Univariable and multivariable Cox proportional hazard regressions for overall survival (OS).

\begin{tabular}{|c|c|c|c|c|c|c|c|c|}
\hline \multirow[b]{2}{*}{ Variable } & \multicolumn{3}{|c|}{ Univariable } & \multicolumn{5}{|c|}{ Multivariable } \\
\hline & HR & $95 \% \mathrm{CI}$ & $p$-Value & HR & $95 \% \mathrm{CI}$ & $p$-Value & $95 \% \mathrm{CI}^{\wedge}$ & $p$-Value ${ }^{\wedge}$ \\
\hline Female gender & 0.85 & $0.63-1.14$ & 0.271 & - & - & - & - & - \\
\hline Age $\geq 65$ years & 1.23 & $0.93-1.61$ & 0.142 & - & - & - & - & - \\
\hline Time to first-line TKI* $<12$ months & 1.61 & $1.23-2.10$ & $<0.001$ & 1.04 & $0.74-1.45$ & 0.837 & $0.69-1.56$ & 0.865 \\
\hline KPS $<80 \%$ & 7.02 & $3.53-13.98$ & $<0.001$ & 3.48 & $1.36-8.93$ & 0.009 & $1.20-10.15$ & 0.022 \\
\hline Non-clear cell histology & 1.18 & $0.68-2.07$ & 0.557 & - & - & - & - & - \\
\hline Sarcomatoid features & 1.75 & $1.01-3.01$ & 0.045 & 1.38 & $0.75-2.53$ & 0.307 & $0.71-2.68$ & 0.350 \\
\hline Fuhrman grade 3-4 & 2.02 & $1.52-2.68$ & $<0.001$ & 1.57 & $1.13-2.19$ & 0.007 & $1.05-2.35$ & 0.029 \\
\hline Prior immunotherapy & 0.89 & $0.58-1.37$ & 0.593 & - & - & - & - & - \\
\hline Number of metastatic sites $>2$ & 2.37 & $1.81-3.12$ & $<0.001$ & 1.26 & $0.82-1.95$ & 0.296 & $0.75-2.11$ & 0.379 \\
\hline Local recurrence & 1.46 & $1.07-1.98$ & 0.017 & 1.08 & $0.74-1.59$ & 0.689 & $0.69-1.69$ & 0.729 \\
\hline Retroperitoneal/mediastinal lymph node metastases & 1.84 & $1.40-2.40$ & $<0.001$ & 1.36 & $0.97-1.90$ & 0.072 & $0.92-2.00$ & 0.119 \\
\hline Lung metastases & 1.77 & $1.29-2.42$ & $<0.001$ & 1.68 & $1.16-2.42$ & 0.006 & $1.06-2.65$ & 0.026 \\
\hline Liver metastases & 1.17 & $0.85-1.61$ & 0.345 & - & - & - & - & - \\
\hline Pancreas metastases & 0.58 & $0.36-0.92$ & 0.022 & 0.84 & $0.44-1.62$ & 0.603 & $0.37-1.92$ & 0.681 \\
\hline Suprarenal gland metastases & 1.17 & $0.82-1.66$ & 0.396 & - & - & - & - & - \\
\hline Bone metastases & 1.65 & $1.25-2.18$ & $<0.001$ & 1.25 & $0.86-1.82$ & 0.237 & $0.83-1.89$ & 0.285 \\
\hline Contralateral kidney metastases & 0.70 & $0.41-1.18$ & 0.180 & - & - & - & - & - \\
\hline Brain metastases & 3.84 & $2.25-6.55$ & $<0.001$ & 1.71 & $0.89-3.27$ & 0.106 & $0.67-4.32$ & 0.259 \\
\hline Other soft tissue metastases & 1.44 & $1.08-1.93$ & 0.014 & 1.37 & $0.93-2.01$ & 0.109 & $0.86-2.17$ & 0.184 \\
\hline Haemoglobin $<$ LLN & 2.80 & $2.01-3.82$ & $<0.001$ & 2.04 & $1.36-3.06$ & 0.001 & $1.23-3.37$ & 0.006 \\
\hline Neutrophils >ULN & 1.93 & $1.20-3.10$ & 0.006 & 1.11 & $0.63-1.94$ & 0.723 & $0.55-2.21$ & 0.774 \\
\hline Platelets >ULN & 2.19 & $1.54-3.14$ & $<0.001$ & 0.85 & $0.52-1.39$ & 0.517 & $0.45-1.61$ & 0.619 \\
\hline $\mathrm{NLR} \geq 3.6$ & 1.91 & $1.46-2.50$ & $<0.001$ & 1.63 & $1.11-2.37$ & 0.012 & $1.06-2.50$ & 0.027 \\
\hline PLR $\geq 157$ & 2.13 & $1.62-2.81$ & $<0.001$ & 1.45 & $0.99-2.11$ & 0.057 & $0.92-2.26$ & 0.106 \\
\hline Serum LDH $>$ ULN & 1.63 & $1.21-2.19$ & 0.001 & 1.80 & $1.27-2.57$ & 0.001 & $1.19-2.72$ & 0.005 \\
\hline Serum corrected calcium $>$ ULN & 1.79 & $1.21-2.65$ & 0.004 & 1.29 & $0.78-2.14$ & 0.315 & $0.73-2.30$ & 0.381 \\
\hline Serum albumin $<$ LLN & 3.24 & $2.26-4.64$ & $<0.001$ & 2.50 & $1.45-4.33$ & 0.001 & $1.23-5.10$ & 0.011 \\
\hline
\end{tabular}

CI, Confidence interval; HR, hazard ratio; KPS: Karnofsky performance status; LDH, lactate dehydrogenase; LLN, lower limit of normal; NLR, neutrophil-to-lymphocyte ratio; PLR, platelet-to-lymphocyte ratio; TKI, tyrosine kinase inhibitor; ULN, upper limit of normal. *Time from diagnosis

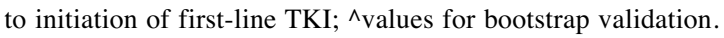


Table III. Multivariable Cox proportional hazard regression for overall survival (05) with the International Metastatic Renal Cell Carcinoma Database Consortium criteria and the pancreatic metastasis (PM) status.

\begin{tabular}{|c|c|c|c|c|c|}
\hline \multirow[b]{2}{*}{ Variable } & \multicolumn{3}{|c|}{ Original dataset } & \multicolumn{2}{|c|}{ Bootstrap validation } \\
\hline & HR & $95 \% \mathrm{CI}$ & $p$-Value & $95 \% \mathrm{CI}$ & $p$-Value \\
\hline KPS $<80 \%$ & 5.28 & $2.57-10.86$ & $<0.001$ & $2.41-11.55$ & $<0.001$ \\
\hline Time to first-line TKI* $<1$ year & 1.38 & $1.04-1.82$ & 0.024 & $1.03-1.83$ & 0.029 \\
\hline Haemoglobin <LLN & 2.26 & $1.63-3.15$ & $<0.001$ & $1.51-3.39$ & $<0.001$ \\
\hline Corrected calcium $>$ ULN & 1.65 & $1.10-2.46$ & 0.015 & $1.01-2.67$ & 0.044 \\
\hline Neutrophil count $>$ ULN & 1.57 & $0.96-2.56$ & 0.070 & $0.83-2.98$ & 0.166 \\
\hline Platelet count $>$ ULN & 1.52 & $1.04-2.22$ & 0.032 & $0.93-2.48$ & 0.096 \\
\hline With PM & 0.66 & $0.40-1.08$ & 0.098 & $0.35-1.23$ & 0.188 \\
\hline
\end{tabular}

CI, Confidence interval; HR, hazard ratio; LLN, lower limit of normal; KPS, Karnofsky performance status; TKI, tyrosine kinase inhibitor; ULN, upper limit of normal. *Time from diagnosis to initiation of first-line TKI.

\section{Discussion}

Despite the improved efficacy of MTT used in clinical practice since 2005 , the prognosis of patients with metastatic RCC remains heterogeneous. As no predictive markers have been discovered for those therapies, the proper identification of baseline features independently influencing survival is essential in routine patient evaluation and fair stratification in prospective interventional trials. Within numerous factors affecting the prognosis, the presence of PM was reported to reduce the probability of death in several studies (10-13). This surprising conclusion contradicts a general concept of cancer, in which greater malignant involvement of the body results in poorer outcomes. However, development of PM usually occurs a long time after the initial diagnosis and, simultaneously, time itself influences on survival when measured between the diagnosis and systemic treatment initiation $(7,14,18)$. Thus, the correlation between these two variables might conceal the explanation of PM phenomenon in metastatic RCC and appeared to be meaningful for the current research.

The present population was similar to those in previous studies. The fraction of patients developing PM was 10\%, which is strictly within the range of $2-11 \%$ reported in the literature (14). The median time to PM development was almost 10 years from initial diagnosis and was peculiar to this organ. In line with other reports, patients in the PM group had a longer interval from diagnosis to TKI initiation (12), greater number of metastatic sites (12), more frequent contralateral kidney metastases $(11,19)$ and favourable risk group assignment (10-12), but also less frequent Fuhrman grade 34 and bone metastases (10) than patients in the non-PM group. The median OS of patients with PM was 46.1 months and was about twice as long as the median OS of patients without PM. Such difference in survivals was even larger than in the recent studies. For example, Grassi et al. reported median OS of 39 months for the PM group and 23 months for the non-PM group (10), while Karla et al. reported median OS of 39 months and 26 months, respectively (12).

In contrast to previous reports, our research revealed that PM did not independently influence survival in patients with metastatic RCC. The PM status was an insignificant predictor when using the log-rank test with the IPTW adjustment and two different approaches of CPHR. What is more, the second CPHR procedure showed that the interval between diagnosis and treatment initiation remained significant after addition of the PM status to the six IMDC criteria. The interval not only probably explains more variability than PM, but also shares some of the variability explained by PM as these variables are moderately, but significantly correlated. Remarkably, patients in the PM group had a six-fold higher chance of starting therapy at one year or later from diagnosis than patients in the non-PM group. As no other IMDC criteria differed significantly between groups, this presumably translated into more frequent assignment to favourable- and intermediate-risk groups in patients with PM when compared to non-PM patients and, subsequently, contributed to improved survival in the PM cohort. Additionally, the metastatic cascade is not only dependent on genetic and epigenetic alterations within cancer cells, but also involves non-neoplastic stromal cells, immune cells, cancer-associated fibroblasts and extracellular matrix components that contribute to cancer progression, both at local and distant sites (20). The patterns of tissue tropism displayed by tumour cells of various origins are well characterized for bone, lung, and brain but little is known about pancreas (21). Thus, herein we stress the necessity of further molecular studies on tissue samples of patients with PM to help identify the primary roots of favourable prognosis in metastatic RCC. For example, such studies may 
focus on pleiomorphic adenoma gene-like 1 (PLAGL1) protein or special AT-rich sequence binding protein 1 (SATB1) mRNA levels. Because decreased PLAGL1 protein level and up-regulation of $S A T B 1$ mRNA in the primary tumour were found to improve survival of patients with RCC, similar levels of those biomarkers obtained from PM samples could straightforwardly reveal some biological explanation for slowly progressing RCCs $(22,23)$.

Retrospective design, singe-centre experience and lack of molecular investigation are noteworthy limitations of this study. Additionally, because pancreatic metastasectomy may increase survival even in cases of extra-pancreatic disease $(24,25)$, we assigned patients who underwent complete resection of PM prior to TKI initiation to the non-PM group to avoid overestimation of the outcome in the PM cohort. This approach may raise difficulties in comparison with studies on PM populations that were treated with multiple modalities or particularly with surgery alone. However, due to this distinct design, our study becomes the most informative for oncologists who wish to start first-line TKI in patients with PM for whom other therapeutic options are not available.

\section{Conclusion}

Overall, the presence of PM at the start of first-line TKI was not an independent factor of improved survival in patients with metastatic RCC, but rather was an indicator of an indolent course of the disease. The true reason for favourable prognosis of patients with PM probably lies in less aggressive tumour biology which results in delayed metastatic spread and initiation of systemic therapy. Further studies focused on molecular differences between tumours from patients with PM and those without PM might be a straightforward way to confirm this hypothesis.

\section{Conflicts of Interest}

None declared.

\section{References}

1 Ferlay J, Soerjomataram I, Ervik M, Dikshit R, Eser S, Mathers C, Rebelo M, Parkin DM, Forman D and Bray F: GLOBOCAN 2012 v1 .0, Cancer Incidence and Mortality Worldwide: IARC CancerBase No. 11 [Internet]. Lyon, France: International Agency for Research on Cancer; 2013. Available from: http:// globocan.iarc.fr/Pages/fact_sheets_population.aspx, accessed on 02/06/2017.

2 Cancer Facts and Figures 2016. American Cancer Society, 2016. Available from: https://old.cancer.org/acs/groups/content/@ research/documents/document/acspc-047079.pdf, accessed on $02 / 06 / 2017$.

3 Janzen NK, Kim HL, Figlin RA and Belldegrun AS: Surveillance after radical or partial nephrectomy for localized renal cell carcinoma and management of recurrent disease. Urol Clin North Am 30(4): 843-852, 2003.

4 Chin AI, Lam JS, Figlin RA and Belldegrun AS: Surveillance strategies for renal cell carcinoma patients following nephrectomy. Rev Urol 8(1): 1-7, 2006.

5 Escudier B, Porta C, Schmidinger M, Rioux-Leclercq N, Bex A, Khoo V, Gruenvald V and Horwich A: Renal cell carcinoma: ESMO Clinical Practice Guidelines for diagnosis, treatment and follow-up. Ann Oncol 27(suppl 5): v58-68, 2016.

6 National Comprehensive Cancer Network. Kidney Cancer (Version 2.2017). Available from: https://www.nccn.org/ professionals/physician_gls/pdf/kidney.pdf, accessed on 02/06/2017.

7 Heng DY, Xie W, Regan MM, Warren MA, Golshayan AR, Sahi C, Eigl BJ, Ruether JD, Cheng T, North S, Venner P, Knox JJ, Chi KN, Kollmannsberger C, McDermott DF, Oh WK, Atkins MB, Bukowski RM, Rini BI and Choueiri TK: Prognostic factors for overall survival in patients with metastatic renal cell carcinoma treated with vascular endothelial growth factortargeted agents: results from a large, multicenter study. J Clin Oncol 27(34): 5794-5799, 2009.

8 Négrier S, Escudier B, Gomez F, Douillard JY, Ravaud A, Chevreau C, Buclon M, Pérol D and Lasset C: Prognostic factors of survival and rapid progression in 782 patients with metastatic renal carcinomas treated by cytokines: a report from the Groupe Français d'Immunothérapie. Ann Oncol 13(9): 1460-1468, 2002.

9 McKay RR, Kroeger N, Xie W, Lee JL, Knox JJ, Bjarnason GA, MacKenzie MJ, Wood L, Srinivas S, Vaishampayan UN, Rha SY, Pal SK, Donskov F, Tantravahi SK, Rini BI, Heng DY and Choueiri TK: Impact of bone and liver metastases on patients with renal cell carcinoma treated with targeted therapy. Eur Urol 65(3): 577-584, 2014.

10 Grassi P, Verzoni E, Mariani L, De Braud F, Coppa J, Mazzaferro V and Procopio G: Prognostic role of pancreatic metastases from renal cell carcinoma: results from an Italian center. Clin Genitourin Cancer 11(4): 484-488, 2013.

11 Grassi P, Doucet L, Giglione P, Grünwald V, Melichar B, Galli L, De Giorgi U, Sabbatini R, Ortega C, Santoni M, Bamias A, Verzoni E, Derosa L, Studentova H, Pacifici M, Coppa J, Mazzaferro V, de Braud F, Porta C, Escudier B and Procopio G: Clinical impact of pancreatic metastases from renal cell carcinoma: a multicenter retrospective analysis. PLoS One 11(4): $\mathrm{e} 0151662,2016$.

12 Kalra S, Atkinson BJ, Matrana MR, Matin SF, Wood CG, Karam JA, Tamboli P, Sircar K, Rao P, Corn PG, Tannir NM and Jonasch E: Prognosis of patients with metastatic renal cell carcinoma and pancreatic metastases. BJU Int 117(5): 761-765, 2016.

13 Santoni M, Conti A, Porta C, Procopio G, Sternberg CN, Basso U, De Giorgi U, Bracarda S, Rizzo M9, Ortega C, Massari F, Iacovelli R, Derosa L, Masini C, Milella M, Di Lorenzo G, Atzori F, Pagano M, Buti S, De Vivo R, Mosca A, Rossi M, Paglino C, Verzoni E, Cerbone L, Muzzonigro G, Falconi M, Montironi R, Burattini L, Santini D and Cascinu S: Sunitinib, pazopanib or sorafenib for the treatment of patients with late relapsing metastatic renal cell carcinoma. J Urol 193(1): 41-47, 2015.

14 Ballarin R, Spaggiari M, Cautero N, De Ruvo N, Montalti R, Longo C, Pecchi A, Giacobazzi P, De Marco G, D'Amico G, Gerunda GE and Di Benedetto F: Pancreatic metastases from renal cell carcinoma: the state of the art. World J Gastroenterol 17(43): 4747-4756, 2011. 
15 Xie J and Liu C: Adjusted Kaplan-Meier estimator and log-rank test with inverse probability of treatment weighting for survival data. Stat Med 24(20): 3089-3110, 2005.

16 Harrell FE Jr., Lee KL and Mark DB: Multivariable prognostic models: issues in developing models, evaluating assumptions and adequacy, and measuring and reducing errors. Stat Med 15(4): 361-387, 1996.

17 Schemper M and Smith TL: A note on quantifying follow-up in studies of failure time. Control Clin Trials 17(4): 343-346, 1996.

18 Motzer RJ, Bacik J, Murphy BA, Russo P and Mazumdar M: Interferon-alpha as a comparative treatment for clinical trials of new therapies against advanced renal cell carcinoma. J Clin Oncol 20(1): 289-296, 2002.

19 Yuasa T, Inoshita N, Saiura A, Yamamoto S, Urakami S, Masuda H, Fujii Y, Fukui I, Ishikawa Y and Yonese J: Clinical outcome of patients with pancreatic metastases from renal cell cancer. BMC Cancer 15: 46, 2015.

20 Azevedo AS, Follain G, Patthabhiraman S, Harlepp S and Goetz JG: Metastasis of circulating tumor cells: favorable soil or suitable biomechanics, or both? Cell Adh Migr 9(5): 345-356, 2015.

21 Jin $\mathrm{K}$, Li T, van Dam H, Zhou F and Zhang L: Molecular insights into tumour metastasis: tracing the dominant events. J Pathol 241(5): 567-577, 2017.
22 Godlewski J, Krazinski BE, Kowalczyk AE, Kiewisz J, Kiezun J, Kwiatkowski P, Sliwinska-Jewsiewicka A, Maslowski Z and Kmiec Z: PLAGL1 (ZAC1/LOT1) expression in clear cell renal cell carcinoma: correlations with disease progression and unfavorable prognosis. Anticancer Res 36(2): 617-624, 2016.

23 Kowalczyk AE, Krazinski BE, Godlewski J, Grzegrzolka J, Kiewisz J, Kwiatkowski P, Sliwinska-Jewsiewicka A, Dziegiel P and Kmiec Z: SATB1 is down-regulated in clear cell renal cell carcinoma and correlates with miR-21-5p overexpression and poor prognosis. Cancer Genomics Proteomics 13(3): 209-217, 2016.

24 Sohn TA, Yeo CJ, Cameron JL, Nakeeb A and Lillemoe KD: Renal cell carcinoma metastatic to the pancreas: results of surgical management. J Gastrointest Surg 5(4): 346-351, 2001.

25 Schwarz L, Sauvanet A, Regenet N, Mabrut JY, Gigot JF, Housseau E, Millat B, Ouaissi M, Gayet B, Fuks D and Tuech JJ: Long-term survival after pancreatic resection for renal cell carcinoma metastasis. Ann Surg Oncol 21(12): 4007-4013, 2014.

Received October 2, 2017

Revised October 21, 2017

Accepted October 25, 2017 